\title{
Effectiveness of Single Platelet-rich Plasma Injection and Rehabilitation in the Management of Calcaneocuboid Syndrome: A Case Series
}

\author{
Vikram Rao ${ }^{1}$, Karthik Anand ${ }^{2}$, Parthiban Jaganathan ${ }^{3}$, Prakash Ayyadurai ${ }^{4}$, Suresh Perumal ${ }^{5}$, Thiagarajan Alwar ${ }^{6}$, \\ Arumugam Sivaraman ${ }^{7}$
}

\begin{abstract}
Background: Calcaneocuboid syndrome is a common yet underdiagnosed condition. It has been noted as a FADI scores complication of plantar flexion and inversion ankle injuries which are one of the most common athletic injuries. It is common in ballet dancers and athletes. Presentation of this condition is lateral foot pain which is exaggerated on inversion.

Aim and objectives: To determine the outcomes of a single platelet-rich plasma (PRP) injection combined with rehabilitation for calcaneocuboid syndrome.

Materials and methods: Six patients ( 3 non-sportsperson, 2 footballers, 1 basketball player) who presented with complaints of chronic lateral ankle pain were evaluated clinically and radiologically and identified to have calcaneocuboid syndrome. Ultrasound-guided PRP injection was done. Preinjection and one and three months postinjection visual analog scale (VAS) and foot and ankle disability index (FADI) scores were noted. They also underwent a rehabilitation program.

Results: One patient was lost to follow-up after 1 month. Five patients showed significant improvement in VAS and FADI at 3 months.

Discussion: The use of PRP has shown proven benefits in the literature. Our study combined PRP along with a rehabilitation program for the subjects.

Conclusion: Single PRP injection along with a rehabilitation program has shown to be effective in the management of calcaneocuboid syndrome. Keywords: Ankle injury, Calcaneocuboid joint, Foot injury, Inversion injury, PRP injection.

Journal of Foot and Ankle Surgery (Asia Pacific) (2021): 10.5005/jp-journals-10040-1136
\end{abstract}

\section{BACKGROUND}

Calcaneocuboid syndrome is a common yet underdiagnosed condition. It has been noted as a complication of plantar flexion and inversion ankle injuries which are one of the most common athletic injuries. It is common in ballet dancers and athletes. Presentation of this condition is lateral foot pain which is exaggerated on inversion. Lateral ankle ligament injuries are commonly addressed, whereas calcaneocuboid injuries may be overlooked. Residual pain following multiple episodes of ankle sprain points toward the diagnosis of calcaneocuboid syndrome. Differential diagnoses include osteochondritis dissecans of the anterolateral aspect of talus, fractures of the cuboid, sinus tarsi syndrome, peroneal injuries, lateral plantar nerve entrapment, os peroneum, Lisfranc injuries, exertional compartment syndrome, and tarsal coalition.

When not addressed early, this condition can lead to chronic debilitating ankle pain and reduce sports performance in athletes. Proper identification and management is essential in reducing loss of on-field time.

The use of autologous platelet-rich plasma (PRP) has been shown to augment the healing of various musculoskeletal injuries. Platelet-rich plasma injections are being routinely used in conditions, such as rotator cuff tears, osteoarthritis of the knees, ulnar collateral ligament tears, lateral epicondylitis, hamstring injuries, and Achilles tendinopathy. The sparse vascularization of the ligaments results in an inadequate release of growth factors for tissue healing. Hence, the use of PRP, which is a source of these growth factors, has been purported to improve the healing process.
${ }^{1-7}$ Department of Arthroscopy and Sports Medicine, Centre for Sports Science, Sri Ramachandra Institute of Higher Education and Research, Chennai, Tamil Nadu, India

Corresponding Author: Vikram Rao, Department of Arthroscopy and Sports Medicine, Centre for Sports Science, Sri Ramachandra Institute of Higher Education and Research, Chennai, Tamil Nadu, India, Phone: +918939724892, e-mail: vikram.rao24@yahoo.in

How to cite this article: Rao V, Anand K, Jaganathan P, et al. Effectiveness of Single Platelet-rich Plasma Injection and Rehabilitation in the Management of Calcaneocuboid Syndrome: A Case Series. J Foot Ankle Surg (Asia Pacific) 2021;8(1):16-18.

Source of support: Nil

Conflict of interest: None

\section{Aim and Objective}

To determine the outcomes of a single PRP injection combined with rehabilitation for calcaneocuboid syndrome.

\section{Materials and Methods}

Patients who presented to the department of arthroscopy and sports medicine with complaints of lateral ankle pain were evaluated. Imaging was performed to rule out fractures. Six patients (three non-sportsperson, two footballers, one basketball player) who presented with complaints of chronic lateral ankle pain were evaluated clinically and radiologically, and identified to have calcaneocuboid syndrome. Twenty milliliters of venous blood 
were withdrawn from the subjects and $2 \mathrm{~mL}$ of PRP was prepared using double-spin technique. After an initial local anesthetic injection to confirm the diagnosis, a single sitting of ultrasoundguided PRP injection was given at the calcaneocuboid joint with the help of a senior musculoskeletal radiologist. Informed consent was obtained. Pre- and postinjection visual analog scale (VAS) was recorded. Following the PRP injection, the individuals underwent a rehabilitation program as suggested by Sampson et al. ${ }^{1}$ Foot and ankle disability index (FADI) scoring was performed at 1 and 3 months following the procedure (Figs 1 and 2).

\section{Results}

All the patients underwent the injection procedure and rehabilitation exercises were taught. All the patients had a good compliance for the first month of rehabilitation. Two athletes voluntarily chose to perform supervised rehabilitation at our center and continued for 2 months.

One patient was lost to follow-up after 1 month. Five patients showed significant improvement in VAS and FADI at 3 months.

\section{Discussion}

\section{Anatomy}

The calcaneocuboid joint is one of the two moveable joints in the transverse tarsal joint complex. It rotates about a medially positioned axis. $^{2}$ The four ligaments connecting the calcaneus and cuboid are the medial calcaneocuboid, dorsolateral calcaneocuboid ligament, short and long plantar calcaneocuboid ligaments. ${ }^{3}$ These ligaments help in reinforcing the weak joint capsule. Cadaveric studies have shown a wide variety of configurations in shape, number, and variety of the calcaneocuboid ligaments. Hence, the term dorsolateral ligament complex has been suggested. ${ }^{4}$

\section{Calcaneocuboid Syndrome}

This condition is also known as locked cuboid, calcaneocuboid syndrome fault syndrome, subluxated cuboid or lateral plantar neuritis. It can occur following an inversion injury of the foot and with repeated lateral ankle sprains and overuse syndrome. Other factors that are likely associated with is syndrome are pronated foot structure, improperly constructed orthoses, uneven running terrain, increased speed work, and faulty shoe construction. There could be a disruption in the structural congruity of the joint which

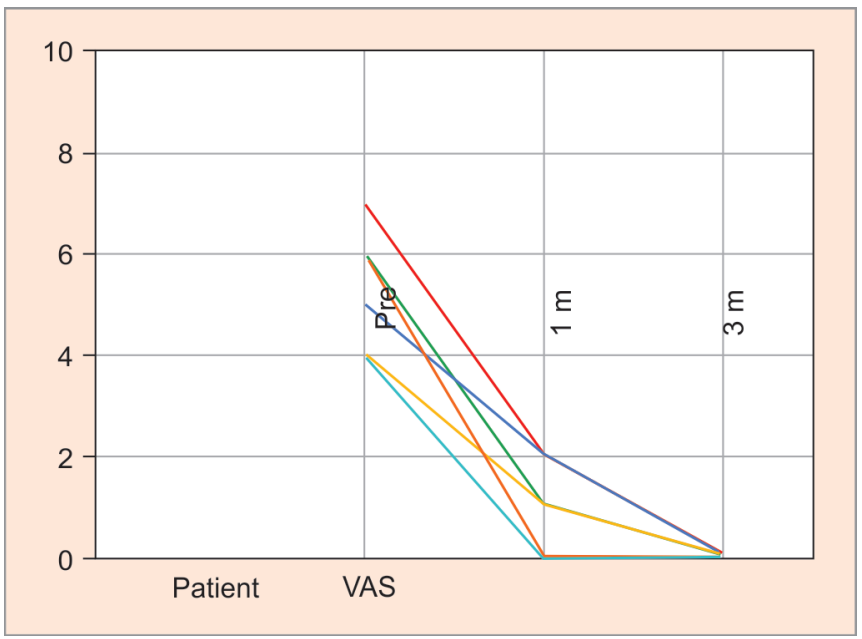

Fig. 1: Visual analog scale (VAS) may in turn irritate the soft tissues surrounding it. ${ }^{5}$ The cuboid acts as a fulcrum for the peroneus longus pulley device. The muscle can pull the lateral aspect of the cuboid dorsally and the medial aspect into a plantar position causing it to become locked and lacking normal range of motion. ${ }^{6}$ The athlete may present with pain over the lateral aspect of the foot, antalgic gait with increased pain during the push-off phase. Midtarsal mobility testing in supination and adduction produces distraction forces and may reproduce the pain. ${ }^{5}$ Diagnosis of the condition is primarily based on history and clinical examination. Imaging is usually required to rule out other differential diagnoses.

The various treatment options mentioned in the literature include manipulation techniques, low-dye taping, orthotics, cuboid pad, stretching, and modalities. A specific manipulation technique has been described by Newell and Woodle. ${ }^{6}$ It is thought that this manipulation realigns the calcaneocuboid joint to its normal position but this theory has not been confirmed.

\section{Platelet-rich Plasma}

It is a product derived from the autologous blood that is greater in platelet concentration compared to the whole blood. It is rich in growth factors; therefore, augmentation of the healing process is the basic theory behind the use of PRP. It also contains cytokines, proteins, fibrinogen, fibrin, and white blood cells, but PRP is consistently defined only by the absolute quantity of platelets and not by other components. ${ }^{7}$ The first use of PRP was in oromaxillofacial surgery in the $1950 \mathrm{~s}^{8}$ It is being currently used in musculoskeletal conditions, such as rotator cuff tears, knee osteoarthritis, lateral epicondylitis, plantar fasciitis, Achilles tendinopathy, hamstring, and quadriceps injuries.

\section{Processing Method}

Different systems are available in the market to obtain PRP, varying from simple centrifugation process to automated process. The automated process seems to lead to more accuracy and allows for more reproducible concentrations. The volume of blood withdrawn depends upon the amount of PRP required. As the volume of whole blood increases, a higher volume of PRP at a higher concentration is available. They may also vary in the amount of leukocytes, erythrocytes, and anabolic and catabolic growth factors. With the use of a cell-separator system, we can obtain a leukocyte poor PRP when large inflammation is not desired.

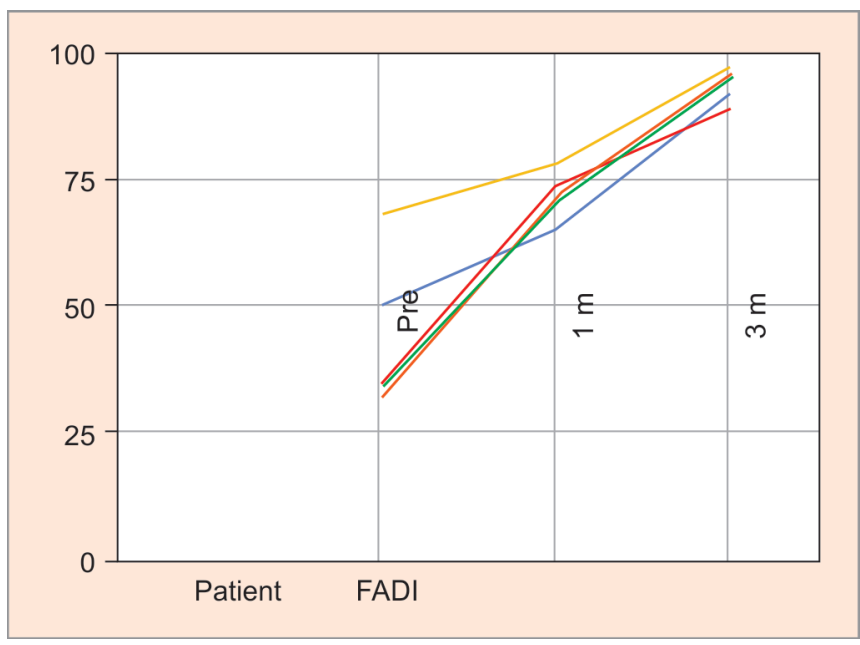

Fig. 2: Foot and ankle disability index (FADI) score 


\section{Ultrasound Guidance}

With the wide use of musculoskeletal injection procedures, it is prudent to utilize the ultrasound for a safe and precise technique. More accurate and efficacious outcomes may be achieved, along with several other benefits, with the use of ultrasound. ${ }^{9}$ In our study, we used ultrasound guidance, done by a senior musculoskeletal radiologist, while injecting the PRP.

\section{Literature}

The use of PRP has become common in clinical practice for its advantages of being easy to use, relative availability, low side effects, and tolerability. Positive outcomes in the form of decreased pain, increased activity, improve. ${ }^{10}$ A review of paper on PRP in orthopedic sports medicine by Mlynarek et al. ${ }^{11}$ states that the vast majority of published literature is level 3 and 4 evidence. Some level 1 evidence studies are also available showing the benefits of PRP injection. ${ }^{12}$ A clear indication for the use of PRP is not easy to be established as evidenced from the available data and is still evolving. Scarce homogeneity of the study population and the variability in the use of preparatory procedures, cell concentration, and dosing of the injection demands the need for more clinical trials in this field. With regard to foot and ankle injuries and procedures, PRP has been used in Achilles pathology, plantar fasciitis, osteochondral lesions, and syndesmotic fusion of ankle. ${ }^{13}$ The use of PRP in the management of calcaneocuboid syndrome could not be found in the available published literature to the best of our knowledge.

\section{Conclusion}

Single PRP injection along with a rehabilitation program has shown to be effective in the management of calcaneocuboid syndrome. Our study incorporated both the interventions, PRP and rehabilitation, and does not reveal whether the PRP injection accelerated the rehabilitation of the athletes. Hence, future studies are required to compare the effectiveness of PRP injection with a control group.

\section{References}

1. Sampson S, Gerhardt M, Mandelbaum B. Platelet rich plasma injection grafts for musculoskeletal injuries: a review. Curr Rev Musculoskelet Med 2008;1(3-4):165-174. DOI: 10.1007/s12178-008-9032-5.

2. Patterson SM. Cuboid syndrome: a review of the literature. J Sports Sci Med 2006;5(4):597-606.

3. Melão L, Canella C, Weber M, et al. Ligaments of the transverse tarsal joint complex: MRI-anatomic correlation in cadavers. Am J Roentgenol 2009;193(3):662-671. DOI: 10.2214/AJR.08.2084.

4. Dorn-Lange NV, Nauck T, Lohrer H, et al. Morphology of the dorsal and lateral calcaneocuboid ligaments. Foot Ankle Int 2008;29(9):942-949. DOI: 10.3113/FAI.2008.0942.

5. Jennings J, Davies GJ. Treatment of cuboid syndrome secondary to lateral ankle sprains: a case series. J Orthop Sports Phys Ther 2005;35(7):7. DOI: 10.2519/jospt.2005.35.7.409.

6. Newell SG, Woodle A. Cuboid syndrome. Phys Sportsmed 1981;9(4):71-76. DOI: 10.1080/00913847.1981.11711057.

7. Maffulli N. Platelet rich plasma in musculoskeletal practice. New York, NY: Springer Berlin Heidelberg; 2016.

8. Marx RE. Platelet-rich plasma: evidence to support its use. J Oral Maxillofac Surg 2004;62(4):489-496. DOI: 10.1016/j.joms.2003.12.003.

9. Özçakar L, Onat \$̧, Gürçay E, et al. Are blind injections ethical or historical? Think twice with ultrasound. Am J Phys Med Rehabil 2016;95(2):158-160. DOI: 10.1097/PHM.0000000000000377.

10. Soomekh DJ. Using platelet-rich plasma in the foot and ankle. Foot Ankle Spec 2010;3(2):88-90. DOI: 10.1177/1938640010364483.

11. MlynarekRA, Kuhn AW, Bedi A. Platelet-rich plasma (PRP) in orthopedic sports medicine. Am J Orthop (Belle Mead NJ) 2016;45(5):290-326.

12. Dallari D, Stagni C, Rani N, et al. Ultrasound-guided injection of plateletrich plasma and hyaluronic acid, separately and in combination, for hip osteoarthritis: a randomized controlled study. Am J Sports Med 2016;44(3):664-671. DOI: 10.1177/0363546515620383.

13. Vannini F, Di Matteo B, Filardo G, et al. Platelet-rich plasma for foot and ankle pathologies: a systematic review. Foot Ankle Surg 2014;20(1):2-9. DOI: 10.1016/j.fas.2013.08.001. 